'División de Pediatría, Unidad de Endocrinología, Escuela de Medicina, Pontificia Universidad Católica de Chile.

2Programa de Doctorado,

Escuela de Salud Pública, Facultad de Medicina, Universidad de Chile. ${ }^{3}$ División de Pediatría, Unidad de Nefrología, Escuela de Medicina, Pontificia Universidad Católica de Chile. ${ }^{4}$ Departamento Pediatría, Escuela de Medicina, Universidad de los Andes de Chile.

Departamento Salud Pública, Escuela de Medicina, Universidad de los Andes de Chile. aBioestadístico.

Proyecto financiado por Universidad de los Andes: Proyecto Med 4, 2006.

Recibido el 30 de noviembre de 2010, aceptado el 11 de mayo de 2011.

Correspondencia a: Dr. Hernán García B.

Lira 85, 5to piso, Santiago Casilla de Correo: 8330074, Santiago, Chile.

Tel: (56-2) 3543402

Fax: (56-2) 6384307

E-mail: hgarciab@med. puc.cl

\section{Prevalencia de hipertensión arterial y su asociación con la obesidad en edad pediátrica}

\author{
RODRIGO BANCALARI ${ }^{1}$, CARLOS DÍAZ ${ }^{2}$, ALEJANDRO MARTÍNEZ-AGUAYO', \\ MARLENE AGLONY ${ }^{3}$, JUANITA ZAMORANO ${ }^{4}$, VERÓNICA CERDA ${ }^{4}$, \\ MANUEL FERNÁNDEZ ${ }^{4}$, FLABIA GARBIN ${ }^{4}$, GABRIEL CAVADA ${ }^{5, a}$, \\ MARÍA VALENZUELA ${ }^{5}$, HERNÁN GARCÍA ${ }^{1}$
}

\section{Prevalence of hypertension in school age children and its association with obesity}

\begin{abstract}
Background: Hypertension in children is a frequently overlooked problem that is an important cardiovascular risk factor. Aim: To determine the prevalence of hypertension among school age children. Material and Methods: Cross-sectional study of 2980 children aged $10 \pm 2$ years (48\% females) from 10 schools of middle and lower class in Metropolitan Santiago. Blood pressure (BP) was measured in the sitting position on three occasions after a rest period, using a mercury sphygmomanometer with appropriate cuff arm diameter, averaging the results of the measurements. Systolic and diastolic hypertension were defined as blood pressure values over 95 percentile for age, sex and height. Results: The overall prevalence of hypertension was $12.2 \%$ in women and $15 \%$ in men $(p<0.05)$. According to nutritional status, the prevalence was 6.7, 8.9, 13.6 and $26 \%$ in underweight, eutrophic, overweight and obese children, respectively $(p<0.01)$. Compared with normal weight children, the risk of being hypertensive for overweight children was 1.6 (95\% confidence intervals (CI) 1.2-2.3) and for obese children was 3.6 (95\% CI 2.8-4.7). Conclusions: The studied children had a high prevalence of hypertension, that was directly related to a higher body mass index.
\end{abstract}

(Rev Med Chile 2011; 139: 872-879).

Key words: Child; Hypertension; Obesity.
T a hipertensión arterial (HTA) es una de la patologías más frecuentes en la población $26 \%{ }^{1}$.

En Chile, la Encuesta Nacional de Salud del año 2003 encontró una frecuencia de HTA del $33,7 \%{ }^{2}$. Cambios del patrón epidemiológico, como incremento de la obesidad, síndrome metabólico y diabetes tipo 2, proyectan que el año 2025 existirían cerca de 1,5 billones de hipertensos ${ }^{1}$. Es una de las condiciones que más se asocia a morbi-mortalidad y provoca aproximadamente 7 millones de muertes cada año ${ }^{3,4}$.
Un niño hipertenso tiene riesgo de ser un adulto hipertenso ${ }^{5}$. Las complicaciones secundarias a HTA pueden observarse precozmente ${ }^{6}$. Es necesario realizar un diagnóstico oportuno para implementar las estrategias necesarias para modificar la historia natural de esta condición y disminuir la patología cardiovascular ${ }^{7,8}$.

Los adolescentes con sobre-peso y obesidad tienen cinco veces más riesgo de desarrollar $\mathrm{HTA}^{9} \mathrm{y}$ si se consideran sólo sujetos con obesidad el riesgo aumenta a 8,5 veces $^{10}$. La obesidad, intolerancia a la glucosa e HTA en la niñez se asocian con mayor riesgo de mortalidad antes de los 55 años $^{5}$. 
Prevalencia de hipertensión arterial en pediatría - R. Bancalari et al

Este estudio evalúa la prevalencia de HTA en población pediátrica de Santiago de Chile y determina su asociación con estado nutricional.

\section{Materiales y Métodos}

\section{Diseño y muestra}

Se diseñó un estudio de base poblacional y corte transversal para estudiar la prevalencia de HTA en escolares chilenos. Se seleccionó una muestra de 2.980 escolares de 6 a 14 años pertenecientes a 10 comunas de Santiago de estrato socioeconómico medio-bajo cuyo universo fueron todos los escolares pertenecientes a los colegios con educación básica de la Sociedad de Instrucción Primaria (SIP) que cursaban en el año 2006 de primero a octavo básico. Esta institución cuenta con 15 colegios de educación básica. Los establecimientos se encuentran distribuidos aleatoriamente en 10 comunas de Santiago y cuentan con una población escolar de aproximadamente 15.000 alumnos.

La muestra se seleccionó en forma aleatoria simple en 10 de los 15 colegios distribuidos en 9 comunas de la capital. El primer colegio seleccionado se utilizó para realizar el estudio piloto y participo la totalidad del colegio. En los restantes 9 establecimientos se realizó muestreo aleatorio estratificado por sexo y curso, participando 336 alumnos por cada colegio.

Para el cálculo del tamaño muestral el número mínimo de pacientes para estudiar la prevalencia de sobrepeso y obesidad es 1.015 escolares, para estudiar la prevalencia de hipertensión arterial es 444 estudiantes. Este estudio evalúo a 2.980 alumnos.

Los criterios de inclusión fueron estar inscritos en los cursos de primero a octavo básico de los colegios de la SIP y que sus padres o tutores hayan aceptado su participación. Quedaron excluidos aquellos que no fueran autorizados por sus padres o tutores, así como también los que no entregaron su consentimiento. El trabajo se realizó entre marzo y diciembre del año 2006, las evaluaciones antropométricas y de presión arterial se realizaron en el gimnasio de cada colegio.

Se contó con 8 pediatras y 16 internos de medicina capacitados, el examen físico se realizó en módulos de examen simultáneos, durante la jornada escolar.

\section{Evaluación antropométrica}

Se determinó la talla en posición erecta em- pleando un estadiómetro de pared fijo marca SECA ${ }^{\circledR}$, se codificó en centímetros más un decimal, realizándose tres mediciones. El resultado final fue el promedio de las mediciones. Se pesó a los niños descalzos, con ropa interior. Se realizaron tres mediciones con pesa marca SECA ${ }^{\circledR}$ modelo 700 , previamente calibrada mediante la utilización de pesos fijos conocidos, determinándose el peso en kilos con un decimal. El resultado final fue el promedio de las mediciones.

\section{Estado nutricional}

El bajo peso, el sobrepeso y la obesidad se definieron mediante el IMC (peso/talla ${ }^{2}$ ). Se consideró bajo peso un IMC bajo el percentil 10, sobrepeso un IMC entre el percentil 85 y 95 , y obeso un IMC por encima del percentil 95 , los valores obtenidos de IMC se compararon tablas estándares según la edad y el sexo del alumno ${ }^{11}$.

\section{Medición de la presión arterial}

Se utilizó un esfingomanómetro de columna de mercurio marca ALPK $2{ }^{\circledR}$ previamente calibrado, con manguito del tamaño adecuado para la edad y el diámetro del brazo.

Se determinó la PA en tres oportunidades separada por 5 minutos de reposo, entre las 8:30 am y 10:00 am en el gimnasio de cada colegio.

La presión arterial sistólica (PAS) y diastólica (PAD) medida en el escolar se comparó con la tabla estándar para la población de EE.UU. de Norteamérica $^{12}$. Para fines de estudio epidemiológico poblacional, se consideró hipertensión arterial una presión arterial mayor a igual al percentil 95 para el sexo y talla del paciente.

\section{Aspectos éticos}

Este trabajo fue aprobado por el Comité de Ética de Investigación en seres Humanos de la Facultad de Medicina de la Universidad de Chile y por el Comité de Ética de la Universidad de los Andes (año 2006). Se requirió la firma de consentimiento informado del apoderado y la asistencia voluntaria del sujeto. A todos los sujetos se les preguntó verbalmente su asentimiento, aquellos que rechazaban ser evaluados fueron excluidos.

\section{Estadística}

Los resultados fueron expresados en promedios y desviaciones estandar, las prevalencias fueron expresadas en porcentajes. Para evaluar el riesgo 
entre los grupos se utilizó el modelo de regresión logística.

Se usaron test paramétricos para evaluar si las diferencias eran estadísticamente significativas. Para las variables no-paramétricas se uso el test de Kruskal-Wallis. Se consideró un p < 0,05 como estadísticamente significativo. Para el análisis estadístico se utilizo el programa SPSS 15.0 de Windows (SPSS Inc, Chicago, EE.UU).

\section{Resultados}

\section{Características generales}

Se obtuvieron datos de 2.980 niños (48\% eran mujeres). Presentaban un promedio de edad de $10,3 \pm 2,3$ años, talla-puntaje de $\mathrm{z}-0,06 \pm 0,94 \mathrm{e}$ IMC-puntaje $z$ de $0,79 \pm 0,95$ (Tabla 1). Entre las niñas y niños no se observó diferencias estadísticamente significativas en la edad $(10,34 \pm 2,3$ vs $10,36 \pm 2,3 ; \mathrm{p}=0,790)$, talla-puntaje $\mathrm{z}(-0,091 \pm$ $0,93$ vs $-0,039 \pm 0,97 ; p=0,135)$. Sin embargo, las mujeres tenían menor IMC-puntaje $\mathrm{z}$ que los varones $(0,76 \pm 0,95 / v s 0,83 \pm 0,96 ; p=0,041)$.

\section{Estado nutricional}

La prevalencia de sobrepeso fue de $24,4 \%$ (IC $95 \%=22,8-25,9)$ y la de obesidad de $20,7 \%$ (IC $95 \%=19,2-22,1)$. La prevalencia de mal nutrición por exceso (sobrepeso más obesidad) de toda la población estudiada fue de $45,1 \%$ (IC $95 \%=42,0$ $48,2)$. La distribución por estado nutricional fue diferente entre mujeres y hombres (Chi-Cuadrado de Pearson; $\mathrm{p}=0,023$ ) (Tabla 1 ).

\section{Presión arterial}

El promedio de PAS fue $103 \pm 13 \mathrm{~mm}$ de $\mathrm{Hg}$ y de PAD $64 \pm 10 \mathrm{~mm}$ de Hg. La prevalencia de HTA de la población total $(\mathrm{n}=2.980)$ fue $13,6 \%$ (IC 95\% 12,4-14,9). La prevalencia de HTA fue menor en el sexo femenino comparado con el sexo masculino (12,2\%/vs 14,95; respectivamente; $\mathrm{p}=0,02)$.

\section{Asociación entre estado nutricional y presión arterial}

La prevalencia de HTA se incrementa a medida que aumenta el IMC $(\mathrm{p}<0,001)$ (Figura 1$)$. La Tabla 2 muestra la prevalencia de HTA según sexo y estado nutricional. La prevalencia de HTA en los escolares con sobrepeso y obesidad tiende a ser mayor en el sexo masculino. Sin embargo, esta diferencia no alcanza significancia estadística con sus pares de sexo femenino ( $p>0,05)$. No hubo diferencia en la prevalencia de HTA por grupo etáreo en ambos sexos. Si comparamos a los alumnos con malnutrición por exceso vs los alumnos eutróficos, observamos que los alumnos con sobrepeso y obesidad presentan un riesgo significativo de tener HTA, con un OR de 1,62 (IC 95\% = 1,23-2,14) y de 3,60 (IC 95\% = 2,80-4,63) respectivamente, en relación con la condición eutrófica.

Las niñas con sobrepeso tienen un riesgo de 1,33 (IC 95\% = 0,88-2,02) de tener HTA en comparación con las escolares eutróficas. Sin embargo, esta asociación no fue estadísticamente significativa ( $p>0,05)$. Las niñas con obesidad tienen un riesgo de 3,12 (IC 95\% = 2,15-4,53) de tener HTA en comparación con las escolares eutróficas

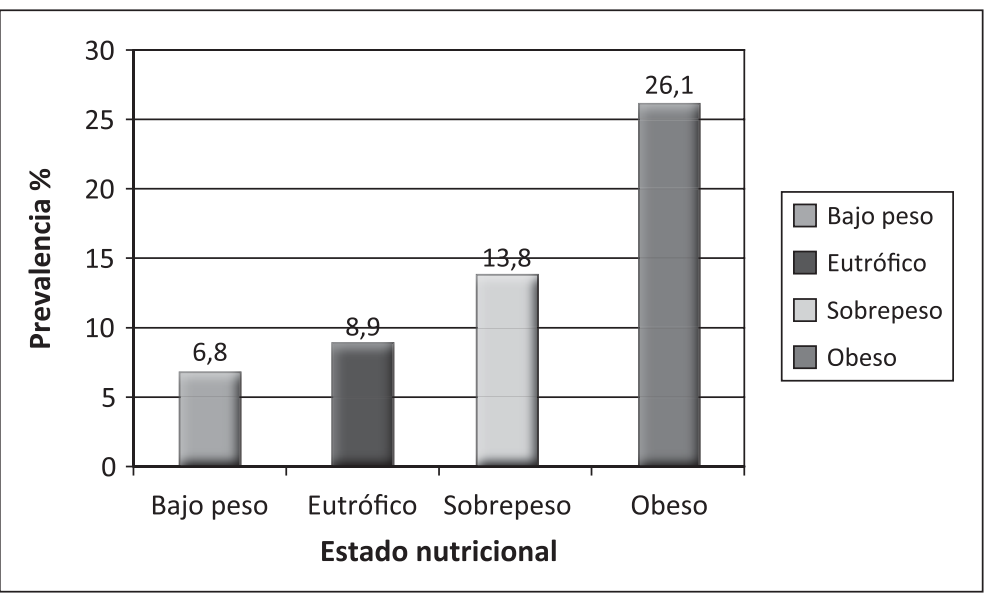

Figura 1. Prevalencia (\%) de HTA (sistólica y/o diastólica) según estado nutricional. 


\begin{tabular}{|c|c|c|c|c|c|c|c|c|}
\hline & $\begin{array}{l}\overline{\text { Jँ }} \\
\stackrel{0}{\circ}\end{array}$ & 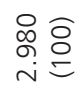 & $\stackrel{m}{\circ}+1 \stackrel{m}{\sim}$ & 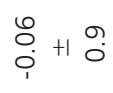 & $\frac{2}{\pi}+1-$ & $\stackrel{m}{\underline{O}}+1 \stackrel{m}{-}$ & 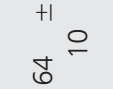 & 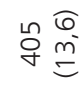 \\
\hline & 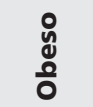 & 총 & $\stackrel{\infty}{\sigma}+1 \stackrel{+}{\sim}$ & $\bar{m}_{0}+1 \stackrel{\circ}{\circ}$ & $\stackrel{\leftrightarrow}{r}+1 \stackrel{m}{\circ}$ & $\stackrel{\infty}{\circ}+1 \stackrel{m}{-}$ & $\otimes_{0}^{\infty}+1=$ & $\underset{\leftarrow}{\stackrel{0}{Q}}$ \\
\hline 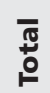 & 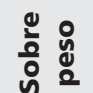 & 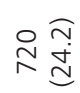 & 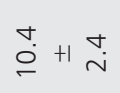 & 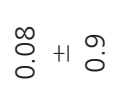 & $\stackrel{\sim}{m}+1 \stackrel{\sim}{0}$ & 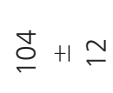 & $\stackrel{2}{6}+1 ㅇ$ & 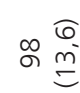 \\
\hline & 递 & 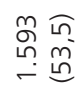 & 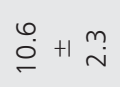 & 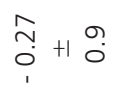 & $\frac{\infty}{\circ}+1 \stackrel{0}{0}$ & $\stackrel{8}{8}+1 \simeq$ & $\hat{\theta}+1$ & 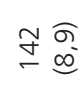 \\
\hline & 일 & 拧 $\stackrel{\widehat{n}}{=}$ & $\stackrel{\bullet}{O}+1 \stackrel{\sim}{\sim}$ & 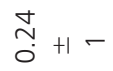 & 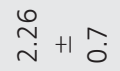 & $\hat{n}+1 \stackrel{0}{\circ}$ & $\bar{\sigma}+\sigma$ & $m \underset{\sigma}{\mathbb{E}}$ \\
\hline & $\begin{array}{l}\bar{\pi} \\
\text { 。ํ }\end{array}$ & $\begin{array}{l}\text { fo } \\
\stackrel{\circ}{\circ} \text { 응 }\end{array}$ & 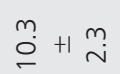 & $\stackrel{m}{0}+1-$ & $\begin{array}{c}0 \\
\infty \\
0\end{array}+1-$ & 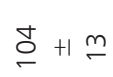 & 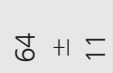 & 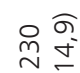 \\
\hline & ơ & $\stackrel{\widetilde{N}}{\stackrel{\widetilde{F}}{\mathbb{N}}}$ & $\hat{a}+1 \stackrel{\sim}{N}$ & $\stackrel{\vec{m}}{m}+1 \stackrel{?}{0}$ & o़ $+1 \stackrel{m}{0}$ & $\stackrel{g}{\circ}+1 \stackrel{m}{n}$ & $\ddot{\infty}+1=$ & হ) \\
\hline 竝 & 흥 & 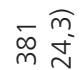 & $\stackrel{+}{\stackrel{\sim}{\circ}+} \stackrel{+}{\sim}$ & $\frac{0}{0}+1-$ & $\stackrel{m}{m}+1 \stackrel{\sim}{0}$ & $\stackrel{\llcorner}{\circ}+1 \stackrel{m}{\underline{n}}$ & $\stackrel{n}{6}+1 ㅇ$ & 요ํ 点 \\
\hline & 题 & 奇会 & $\stackrel{\stackrel{0}{\circ}}{\circ}+\stackrel{m}{\sim}$ & 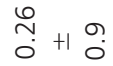 & $\stackrel{n}{\frac{n}{0}}+1 \stackrel{0}{0}$ & $\overline{0}+1 \simeq$ & $\tilde{\theta}_{+1} \circ$ & $P^{\stackrel{0}{\infty}}{ }_{\infty^{0}}$ \\
\hline & 욝 & 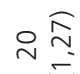 & $\stackrel{\bullet}{\circ}+1 \sim$ & $\stackrel{\infty}{\circ}+1-$ & $\stackrel{\frac{0}{i}}{\stackrel{1}{*}}+\stackrel{6}{0}$ & $\hat{n}+1 \stackrel{0}{ }$ & $8+1=$ & $\sim \stackrel{\widehat{O}}{=}$ \\
\hline & $\begin{array}{l}\overline{0} \\
\stackrel{0}{0}\end{array}$ & $\stackrel{m}{\stackrel{m}{\sigma}} \stackrel{\partial}{=}$ & $\stackrel{+}{\circ}+\stackrel{+}{\sim}$ & $\stackrel{8}{0}+\stackrel{\sigma}{0}$ & 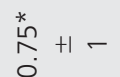 & $\overline{0}+1 \stackrel{\simeq}{\simeq}$ & $\underset{6}{+}+1$ 으 & $\stackrel{*}{\stackrel{N}{N}}$ \\
\hline & : & $\stackrel{\stackrel{0}{\infty}}{\stackrel{\infty}{\infty}} \stackrel{0}{=}$ & $\stackrel{g}{a}+\stackrel{m}{i}$ & 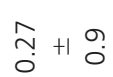 & $\stackrel{n}{\sigma}+1 \stackrel{\sim}{\sigma}$ & 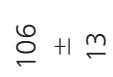 & $\hat{6}+1=$ & $\hat{n} \underset{\sim}{\stackrel{m}{\sim}}$ \\
\hline हू & 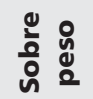 & 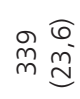 & $\stackrel{+}{\circ}+\underset{\sim}{\sim}$ & $\begin{array}{l}+1 \\
\stackrel{+1}{0} \\
0 \\
0\end{array}$ & $\begin{array}{l}+1 \\
\stackrel{+1}{\sim} \sim \\
\end{array}$ & $\stackrel{m}{\stackrel{\theta}{\theta}}+1=$ & $\Xi+1 \sigma$ & $\stackrel{\text { m }}{\stackrel{\frac{\pi n}{E}}{=}}$ \\
\hline & 爮 & 尽尽 & $\stackrel{n}{\circ}+\stackrel{+}{\sim}$ & 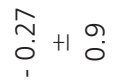 & 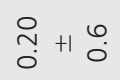 & g $+1 \simeq$ & $\tilde{6}+1 \stackrel{0}{0}$ & $\approx \widehat{g}$ \\
\hline & 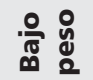 & $\stackrel{\stackrel{\sim}{\sim}}{\stackrel{\mathcal{F}}{E}}$ & 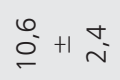 & $\begin{array}{l}0 \\
0 \\
0 \\
i\end{array}+1-$ & 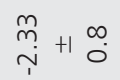 & $\hat{a}+1=$ & $\widetilde{\sigma}+1 \infty$ & $-\widehat{\Xi}$ \\
\hline 递 & 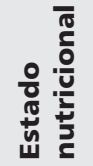 & $\frac{\widehat{o}}{\varepsilon^{\circ}}$ & $\begin{array}{l}\tilde{0} \\
i \frac{c}{\pi} \\
\dot{0} \\
\dot{0} \\
\tilde{0} \\
\end{array}$ & $\begin{array}{l}\frac{0}{90} \\
\frac{0}{5} \\
\frac{2}{2} \\
\frac{0}{0} \\
\frac{0}{10}\end{array}$ & 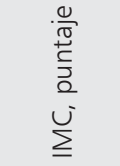 & 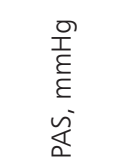 & 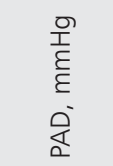 & 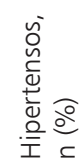 \\
\hline
\end{tabular}


Tabla 2. Distribución de la muestra según estado nutricional y prevalencia de HTA, según sexo

\begin{tabular}{|lcccccc|}
\hline & \multicolumn{2}{c}{ Femenino } & \multicolumn{2}{c}{ Masculino } & \multicolumn{2}{c|}{ Total } \\
E. nutricional & $\%$ & $\mathbf{I C 9 5 \%}$ & $\mathbf{\%}$ & $\mathbf{I C 9 5 \%}$ & $\mathbf{\%}$ & $\mathbf{I C 9 5 \%}$ \\
\hline Bajo peso & 4,0 & $0,1-20$ & 10,0 & $13,0-33,1$ & 6,8 & $1,4-18,6$ \\
\hline Eutrófico & 8,9 & $7,0-11,2$ & 8,9 & $7,0-11,1$ & 8,9 & $7,5-10,4$ \\
\hline Sobrepeso & 11,7 & $8,4-15,6$ & 15,6 & $12,1-19,7$ & 13,8 & $11,3-16,5$ \\
\hline Obeso & 23,6 & $18,6-29,2$ & 28,2 & $23,5-33,3$ & 26,1 & $22,7-29,8$ \\
\hline
\end{tabular}

$(\mathrm{p}<0,001)$. Los varones con sobrepeso y obesidad tienen un riesgo de 1,90 (IC 95\% = 1,31-2,75) y de 4,01 (IC 95\% = 2,85-5,65), respectivamente de tener HTA en comparación con los escolares eutróficos $(\mathrm{p}<0,001$ y $\mathrm{p}<0,001$; respectivamente) (Tabla 3).

\section{Discusión}

En nuestro estudio detectamos una prevalencia de HTA de 13,6\% en la población total. Al analizar los datos según estado nutricional observamos los eutróficos con 8,9\%, sobrepeso $13,8 \%$ y con obesidad 26,1\%.

La HTA es una enfermedad crónica, puede corresponder a hipertensión esencial o ser secundaria a diversas causas, entre las cuales se encuentran enfermedades renales, coartación aórtica o endocrinopatías. En niños las causas secundarias ocurren con mayor frecuencia que en adultos, pero la mayoría de los niños con hipertensión leve a moderada presentan hipertensión esencial ${ }^{13}$.

Existe preocupación que la incidencia de hipertensión esta aumentando junto al incremento de malnutrición por exceso en la población pediátrica $^{12,13}$.

Detectamos una prevalencia de malnutrición por exceso de $45,1 \%, 24,4 \%$ tenían sobrepeso y $20,7 \%$ eran obesos. Estos resultados son consistentes con el incremento de la prevalencia de malnutrición por exceso en los niños chilenos en los últimos años. La Junta Nacional de Auxilio Escolar y Becas reportó el año 2003 una prevalencia de obesidad en niños de primer año básico de 17,2\%, que aumentó a 19,4\% en el año 2006, siendo este último resultado muy similar a lo observado en nuestra serie ${ }^{14,15}$.

El mecanismo mediante el cual la malnutrición
Tabla 3. Riesgo del sobrepeso y la obesidad para tener HTA

\begin{tabular}{|cc|}
\hline Sobrepeso* & Obeso* $^{*}$ \\
$1,33(0,88-2,02)^{1}$ & $3,12(2,15-4,53)^{2}$ \\
$1,90(1,31-2,75)^{2}$ & $4,01(2,85-5,65)^{2}$ \\
$1,62(1,23-2,14)^{2}$ & $3,60(2,80-4,63)^{2}$ \\
\hline
\end{tabular}

Los valores muestran el OR (IC 95\%). * = En referencia a la condición nutricional eutrófica. ${ }^{1}=p>0,05 ;{ }^{2}=p<0,001$.

por exceso puede provocar HTA esta dado por acumulación de grasa visceral, la cual se asocia con insulino-resistencia y aumento de la actividad del sistema renina angiotensina. El hiperinsulinismo secundario a la insulino-resistencia aumenta la reabsorción de sodio a nivel renal y aumenta el tono simpático. Además, estudios experimentales han evaluado que la leptina también aumentaría el tono simpático. Todos estos factores influyen en la elevación de la presión arterial ${ }^{16}$.

Es interesante comparar los hallazgos de nuestro estudio con trabajos realizados en el resto de América. Estudios en México y Brasil muestran prevalencias de HTA de $8,4 \%$ y $5 \%{ }^{17,18}$. En EE.UU. de Norteamérica 4,5\% (en población de raza blanca, afro-americana-hispánicos y asiáticos) ${ }^{19}$.

En Chile, existen escasos estudios de prevalencia de HTA en niños y adolescentes. En el año 1979 se realizó una investigación en 1.391 escolares de 5 a 19 años, pertenecientes a un colegio privado de nivel socioeconómico medio-alto de Santiago. Como resultado se obtuvo que el 11,8\% de la población tenía HTA sistólica y el 5,2\% tenía HTA diastólica (ambos sobre el p95) ${ }^{20}$. En el 2009 Aglony y cols, evaluaron a 112 pacientes escolares chilenos de 6 a 13 años, describieron $2,7 \%$ de hipertensos y $4 \%$ de pre hipertensos ${ }^{21}$. 
Barja y cols detectaron 21\% de HTA sistólica en 71 niños obesos severos ${ }^{22}$. Burrows y cols detectó en niños puberales diferencias estadísticamente significativas entre 45 niños eutróficos y 112 obesos en los valores absolutos de presión arterial ${ }^{23}$. Es importante destacar que la definición de HTA no es la misma en todos los estudios, situación que altera la comparabilidad entre los estudios. Pese a estas diferencias, podemos observar que la malnutrición por exceso constituye un factor de riesgo para tener HTA.

La prevalencia obtenida de HTA en nuestro trabajo es mayor a la observada previamente, esto puede explicarse por la alta prevalencia de malnutrición por exceso en esta población $(45,1 \%)$. De hecho existen estudios con prevalencias de HTA superiores a las nuestras en poblaciones con mayor frecuencia de obesidad ${ }^{24}$.

En Chile, la definición de HTA se basa en la referencia norteamericana ${ }^{12-25}$. Es probable que no sean los parámetros de normalidad ideal para población chilena considerando las diferencias étnicas de las dos poblaciones ${ }^{26}$.

Estudios en población adulta chilena muestran que los Mapuches tendrían prevalencias de HTA mayores que los Aymaras (19,6\% vs 6,9\%) lo que confirma que la etnia juega un rol en la génesis de $\mathrm{HTA}^{27}$.

Al comparar el promedio de presión arterial obtenido en 6 estudios europeos con el promedio de presión arterial de la referencia americana, se encontró una diferencia importante en la presión arterial sistólica y la presión arterial diastólica, siendo ambas mayores en los niños europeos ${ }^{28}$.

Otro aspecto que dificulta la evaluación de la presión arterial en niños, estaría dada a una respuesta fisiológica, caracterizada por una descarga adrenérgica secundaria a la toma de la presión arterial, la cual produce un fenómeno adaptativo que provoca que la medición en un mismo niño en diferentes ocasiones, tienda a disminuir significativamente, con la consecuente variación de la prevalencia de $\mathrm{HTA}^{29,30}$. Sorof y cols ${ }^{19}$ observó que la prevalencia de HTA en niños disminuyó a medida que se realizaban nuevas mediciones de presión arterial, observando una prevalencia de 19,4\%-9,5\%-4,5\% respectivamente con un espacio de tiempo de 10 días entre cada medición. Además, existen otros factores a considerar en las diferencias de prevalencia de HTA y que pueden explicarse por número de tomas de presión arterial, fenómeno de delantal blanco y errores operador dependiente ${ }^{31}$.

Otro factor a considerar es la ingesta diaria de sal, nosotros no realizamos un cuestionario acerca de este tema, pero se estima que en Chile la ingesta promedio de sal en los niños es de 11 gramos al día en contraste con lo sugerido por la organización mundial de la salud que recomienda no ingerir más de 5 gramos al día ${ }^{32}$.

Un estudio de cohorte prospectivo realizado en Chile en 920 personas adultas encontró que luego de 5 años de seguimiento, el riesgo de desarrollar un evento cardiovascular no fatal (infarto agudo de miocardio, angina inestable, accidente vascular encefálico o crisis isquémica transitoria) para la población hipertensa fue 2,49 veces mayor, en comparación con la población sin $\mathrm{HTA}^{33}$. La hipertensión en edad pediátrica ha demostrado ser un factor de riesgo independiente para hipertensión en la edad adulta, y se ha asociado con marcadores tempranos de enfermedad cardiovascular, como hipertrofia de ventrículo izquierdo, ateroesclerosis, disfunción diastólica y también a un mayor riesgo de mortalidad antes de los 55 años en comparación a la población no hipertensa ${ }^{5,13}$.

Din-Dzietham y col ${ }^{34}$, evaluaron la prevalencia de hipertensión arterial en escolares de 8 a 17 años y su asociación con la obesidad. Para esto, los autores hicieron una revisión de los distintos estudios de corte transversal representativos de la población de EE.UU. de Norteamérica y que fueran realizados entre el año 1963 y 2002. Encontraron que a partir del año 1988 la prevalencia de hipertensión arterial se ha incrementado. Al analizar el OR en el último estudio analizado (1999-2002), Encontró que el riesgo de tener hipertensión arterial por cada unidad de incremento en el IMC fue de 2,1 $(\mathrm{IC} 95 \%=1,5-3,0), 1,8(\mathrm{IC} 95 \%=1,2-2,6)$ y 3,2 $($ IC95\% $=2,4-4,4)$ veces mayor, para escolares de raza negra, blanca y mexicanos americanos, respectivamente.

El alto porcentaje de malnutrición por exceso e HTA observado en esta población de Santiago de Chile constituye un importante factor de riesgo cardiovascular. Las características de nuestra muestra no permite extrapolar los resultados a todos los escolares chilenos, pero si a un grupo mayoritario de ellos. Es necesario estudiar la prevalencia de HTA en regiones o grupos de distinto nivel socioeconómico. Desarrollar hábitos de vida saludable y revisar constantemente las prácticas 
de salud son fundamentales para disminuir el desarrollo de patología cardiovascular.

\section{Referencias}

1. Kearney PM, Whelton M, Reynolds K, Muntner P, Whelton PK, He J. Global burden of hypertension: analysis of worldwide data. Lancet 2005; 365: 217-23.

2. Guía Clínica Hipertensión Arterial Primaria o Esencial en personas de 15 años y más. 1ra Ed. Santiago: Minsal, 2006.

3. Yusuf S, Reddy S, Ounpuu S, Anand S. Global burden of cardiovascular diseases: part I-general considerations, the epidemiologic transition, risk factors, and impact of urbanization. Circulation 2001; 104: 2746-53.

4. Ezzati M, López AD, Rodgers A, Vander Hoorn S, Murray CJ; Comparative Risk Assessment Collaborating Group. Selected major risk factors and global and regional burden of disease. Lancet 2002; 360: 1347-60.

5. Franks PW, Hanson RL, Knowler WC, Sievers ML, Bennett PH, Looker HC. Childhood obesity, other cardiovascular risk factors, and premature death. $\mathrm{N}$ Engl J Med 2010; 362.6: 485-93.

6. Daniels SR, Loggie JM, Khoury P, Kimball TR.Left ventricular geometry and severe left ventricular hypertrophy in children and adolescents with essential hypertension. Circulation 1998; 97: 1907-11.

7. Levy D, Garrison RJ, Savage DD, Kannel WB, Castelli WP. Prognostic implications of echocardiographically determined left ventricular mass in the Framingham Heart Study. N Engl J Med 1990; 322: 1561-6.

8. Casale PN, Devereux RB, Milner M, Zullo G, Harshfield GA, Pickering TG, et al. Value of echocardiographic measurement of left ventricular mass in predicting cardiovascular morbid events in hypertensive men. Ann Intern Med 1986; 105: 173-8.

9. Field AE, Cook NR, Gillman MW. Weight status in childhood as a predictor of becoming overweight or hypertensive in early adulthood. Obes Res 2005; 13: 163-9.

10. Srinivasan SR, Bao W, Wattigney WA, Berenson GS. Adolescent overweight is associated with adult overweight and related multiple cardiovascular risk factors: the Bogalusa Heart Study. Metabolism 1996; 45: 235-40.

11. Kuczmarski RJ, Ogden CL, Grummer-Strawn LM, Flegal KM, Guo SS, Wei R, et al. CDC growth charts: United States. Adv Data 2000; 314: 1-27.

12. National High Blood Pressure Education Program Working Group on Hypertension Control in Children and Adolescents. The Fourth Report on the Diagnosis, Evaluation, and Treatment of High Blood Pressure in Children and Adolescents. Pediatrics 2004; 114: 555-76.
13. Hansen ML, Gunn PW, Kaelber DC. Underdiagnosis of Hypertension in Children and Adolescents. JAMA 2007; 298: 874-9.

14. Eyzaguirre F, Mericq V, Ceresa S, Youlton R. Prevalencia de sobrepeso y obesidad en niños que se controlan en pediatría ambulatoria en Clínica Las Condes. Rev Chil Pediatr 2005; 76: 143-9.

15. Sociedad Chilena de Obesidad (SOCHOB), Estadísticas chilenas de obesidad, sobrepeso y patologías metabólicas asociadas en adultos y niños. http://www.sochob.cl, consultadas el 2006.

16. Halpern A, Mancini MC, Magalhães ME, Fisberg M, Radominski R, Bertolami MC, et al. Metabolic syndrome, dyslipidemia and type 2 diabetes in youth: from diagnosis to treatment. Diabetol Metab Syndr 2010; 2: 55.

17. Perichart-Perera O, Balas-Nakash M, Schiffman-Selechnik E, Barbato-Dosal A,Vadillo-Ortega F. Obesity increases metabolic syndrome risk factors in school-aged children from an urban school in Mexico city. J Am Diet Assoc 2007; 107: 81-91.

18. Monego ET, Jardim PC. Determinants of risk of cardiovascular diseases in schoolchildren. Arq Bras Cardiol 2006; 87: 37-45

19. Sorof JM, Lai D, Turner J, Poffenbarger T, Portman RJ. Overweight, ethnicity, and the prevalence of hypertension in school-aged children. Pediatrics 2004; 113: 475 82.

20. Norero C, Vargas N, Mayne D, Fernández C. Alumnos Srs. Monti A., Kutz M. y Cabrera R. Cifras tensionales en poblacion escolar chilena urbana: I. Parte. Rev Chil Pediatr 1980; 51: 184-93.

21. Aglony M, Arnaiz P, Acevedo M, Barja S, Márquez S, Guzmán B, et al. Perfil de presion arterial e historia familiar de hipertensión en niños escolares sanos de Santiago de Chile. Rev Med Chile 2009; 137: 39-45.

22. Barja S, Arteaga A, Acosta A, Hodgson M. Resistencia insulínica y otras expresiones el síndrome metabólico en niños obesos chilenos. Rev Med Chile 2003; 131: 25968.

23. Burrows R, Burgueño M, Leiva L, Ceballos X, Guillier I, Gattas V, et al. Perfil metabólico de riesgo cardiovascular en niños y adolescentes obesos con menor sensibilidad insulínica. Rev Med Chile 2005; 133: 795-804.

24. Urrutia-Rojas X, Egbuchunam CU, Bae S, Menchaca J, Bayona M, Rivers PA, et al. High blood pressure in school children: prevalence and risk factors. BMC Pediatr 2006 16; 6: 32.

25. Lagomarsino E, Saieh C, Aglony M. Recomendación de Ramas: Actualizaciones en el diagnóstico y tratamiento de la Hipertensión Arterial en Pediatría. Rama de Ne- 
Prevalencia de hipertensión arterial en pediatría - R. Bancalari et al

frología, Sociedad Chilena de Pediatría. Rev Chil Pediatr 2008; $79: 63-81$.

26. Chiolero A, Bovet P, Paradis G, Paccaud F. Has blood pressure increased in children in response to the obesity epidemic?. Pediatrics 2007; 119: 544-53.

27. Pérez F, Carrasco E, Santos J, Calvillán M, Albala C. Prevalencia de obesidad, hipertensión arterial y dislipidemia en grupos aborígenes rurales de Chile. Rev Med Chile 1999; 127: 1169-75.

28. De Man SA, Andre JL, Bachmann H, Grobbee DE, Ibsen $\mathrm{KK}$, Laaser U, et al. Blood pressure in childhood: pooled findings of six European studies. J Hypertens 1991; 9: 109-14.

29. Genovesi S, Giussani M, Pieruzzi F, Vigorita F, Arcovio C, Cavuto S, et al. Results of blood pressure screening in a population of school-aged children in the province of Milan: role of overweight. J Hypertens 2005; 23: 493-7.

30. Jackson LV, Thalange NK, Cole TJ. Blood pressure centiles for Great Britain. Arch Dis Child 2007; 92: 298-303.

31. Rose G. Standardisation of observers in blood-pressure measurement. Lancet 1965; 1: 673-4.

32. Saieh C, Lagomarsino E. Arterial Hypertension and salt intake in pediatrics. Rev Chil Pediatr 2009; 80: 11-20.

33. Koch E, Otárola A, Manríquez L, Kirschbaum A, Paredes M, Silva C. Predictors of non fatal cardiovascular events in a Chilean cohort: results of the San Francisco Project. Rev Med Chile 2005; 133: 1002-12.

34. Din-Dzietham R, Liu Y, Bielo MV, Shamsa F. High blood pressure trends in children and adolescents in national surveys, 1963 to 2002. Circulation 2007; 116: 1488-96. 